\title{
Modeling the adult female phantom in the supine and prone postures and initial dose assessment in breast cancer diagnosis with Neutron Stimulated Emission Computed Tomography
}

\author{
N. Araghian, H. Miri-Hakimabad and L. Rafat-Motavalli ${ }^{\star}$ \\ Physics Department, College of Sciences, Ferdowsi University of Mashhad, 91775-1436 Mashhad, Iran.
}

Received 3 June 2014 - Accepted 24 November 2014

\begin{abstract}
In this study, the delivered dose for breast cancer diagnosis by NSECT was assessed in two different configurations, using a model of a human whole body, which was simulated in the supine and prone positions. In the system with the phantom in the prone posture, the adjacent organ doses were considerably decreased. In total irradiation, the breast equivalent dose was less than $10 \mathrm{mSv}$, which is received in a typical chest CT scan. To apply NSECT as a low-dose clinical imaging system, improving the detection system is required.
\end{abstract}

Keywords: NSECT / breast cancer detection / prone and supine positions / dosimetry

\section{Introduction}

Specification of the elemental concentrations is a helpful idea for investigating the human body to diagnose many disorders at very early stages or in progression (Andrási et al., 1993; Garg et al., 1994; Hollán, 1997; Powell et al., 1998; Geraki et al., 2002; Powell, 2005; Yaman et al., 2005; Mutter et al., 2007). This approach can be easily accessible if it is performed in a non-invasive and non-destructive manner. The distinctive aspect lies in providing a three-dimensional map of the elements of the body or part of the body through the spectral tomographic imaging with a low-dose and portable measuring system. All of the above features can be potentially found in a new in vivo spectroscopic imaging technique called Neutron Stimulated Emission Computed Tomography (NSECT) (Kapadia et al., 2008).

NSECT has a potential for a broad range of applications in both medical and biological research. It was suggested that this method could be applied to diagnose thalassemia, Alzheimer's, hemosiderosis, hemochromatosis and also several cancers such as prostate and breast cancer (Kapadia et al., 2008). Breast cancer is a common cancer of global concern, which is easily treated if it is diagnosed before the tumors or calcifications begin to appear. It should be noted that in treating breast cancers, the classification of the abnormalities is necessary to manage the treatment planning. Using NSECT makes it possible to diagnose breast cancer at an early stage and to differentiate between benign and malignant tumors, while other conventional imaging techniques such as CT or mammography suffer from the lack of these two abilities (Kapadia et al., 2006).

\footnotetext{
^ rafat@ferdowsi.um.ac.ir
}

The physical principle of NSECT is based on bombarding the region of interest of the body by fast neutrons and detecting the spectra of the characteristic gamma rays, which are emitted from the de-excitation of the elements' nuclei. The energy of emitted gamma rays is unique, and consequently the type and quantity of elements can be determined by analyzing the gamma spectra. If this procedure is implemented in a tomographic geometry, the spatial distribution of elemental concentration can be imaged.

Due to the in vivo nature of NSECT, the main goal of this method for breast cancer detection is to develop a portable clinical measuring system, which is applicable for controlling the health of individuals and treating patients without any biopsy (Sharma et al., 2006). For this purpose, the dose received by the body should be determined to decrease concerns about risks associated with breast cancer detection by NSECT, especially for healthy individuals. In a preliminary estimation, it was observed that the dose delivered to a simple model of the breast in an initial NSECT acquisition is comparable with the amount of the dose received in a mammogram scan (Sharma et al., 2007a). However, accurate determination of the dose in a comprehensive study is required. In this paper, the dose delivered to the organs in NSECT was assessed using a model of a human wholebody.

\section{Materials and methods}

\subsection{Developing phantoms for breast cancer studies}

The Adult Female (AF) reference computational phantom was used in the current study. This phantom was defined by the 
International Commission on Radiological Protection (ICRP) for dosimetry purposes. According to the ICRP statement in Publication 110 (Grosswendt, 2012), the AF phantom's position is related to the supine posture. Compared with the supine posture, gravity has different effects on the body positioned in the prone posture, so that the breast becomes pendent and the shape of other organs is changed. In addition, when the patient is positioned in the prone posture, the table presses organs located in the abdominal region.

Therefore, to develop two phantoms in the supine and prone postures, a procedure was created to apply some changes in the AF, as described here: a series of tomographic MRI images of a healthy volunteer's breasts in the prone and supine postures was acquired. The axial T2-weighted scan was performed on a Siemens Magnetom MRI system with $1.5 \mathrm{~T}$ static field strength with the following parameters: slice thicknesses of 0.93 and $7.2 \mathrm{~mm}$, number of 176 and 31 planes, and pixel width of 0.64 and $1.19 \mathrm{~mm}$ for scans of the prone and supine postures, respectively. In MRI images, the breasts were segmented into adipose and glandular tissues using image processing and 3D-modeling software: 3D-DOCTOR ${ }^{\mathrm{TM}}$ (Able Software Corp., MA). Due to the difficulty in making any change to the shape and position of the organs and also the position of the body in the voxel phantom (Xu and Eckerman, 2010), the voxel model of the AF was converted into a polygon mesh (PM) in which individual organs and the outer body contour were represented by a large array of triangular surfaces. For some organs such as the breasts, the polygon mesh must be converted into Non-Uniform Rational B-Spline (NURBS) surfaces so that reforming and smoothing the surfaces is possible by using the control points. This process was performed using Rhinoceros ${ }^{\mathrm{TM}}$ (McNeel North America, WA). Then, organs were voxelized using an innovative method developed by our research group and a hybrid voxel phantom was created (Bolch et al., 2010) using the Rhinoceros software (Hoseinian et al., 2014).

The volunteer was selected since she had a similar height, weight and trunk circumference to the AF phantom $(160 \mathrm{~cm}$ in height and 58,000 $\mathrm{g}$ in weight). This similarity helped to adjust the trunk and breasts of the subject to those of the AF, because it provides a good overlapping between their body contours. Therefore, the skin adjacent to the breasts of the AF was replaced by that of subject. In the prone position, the skin adjacent to the breasts of the phantom was rebuilt as a combination of the skin in the back of the AF and the chest of the subject.

However, the mass of the subject's breasts is not equal to the reference quantity, which may arise from the difference in race. Also, the shape of them was different from that of the AF's breasts. The mass of the subject's breasts was calculated as the sum of the mass of glandular and adipose tissues. The mass of each tissue was calculated separately from the volume obtained from the 3D-DOCTOR software weighted by the density values recommended in ICRP Publication 89. Consequently, two phantoms were developed, replacing the AF's breasts with the subject's breasts in the supine and prone postures, separately. In addition, two other phantoms were created by adapting the volume of the subject's breasts with the reference value, in order to compare the effect of reference and non-reference breasts on the dose.
To apply the effect of the patient's table, the abdomen and leg regions of the AF were flattened. This procedure was done by the "cage" tools in the Rhinoceros software. Cage tools provide a way to deform objects using another object's control points. They are used to control a relatively complex object or objects (such as polygon mesh objects) or in other words, for which direct point editing is not practical. Here, it is a boxshaped object, which contains control points in three directions, surrounding the trunk and legs. The control points existing in the front face of the box, i.e. the anterior plane of the body, were modified to convert the outer body contour from the supine to prone posture as well as the abdominal organs.

As a result, two phantoms with reference (Ref-MOB) and non-reference masses of the breasts (nonRef-MOB) in both the supine and prone positions were studied. Sagittal and transverse sections of these hybrid voxel phantoms are demonstrated in Figure 1.

\subsection{Simulating the tomographic system}

The tomographic system of NSECT was simulated in two different geometries. The first is a common geometry (cgeometry) used in breast cancer detection (Kapadia, 2009), which contains a $2.5 \mathrm{MeV}$ mono-energetic beam of neutrons with a circular section of $1 \mathrm{~cm}^{2}$. The neutron beam is irradiated perpendicular to the body. Tomographic acquisition is yielded by concurrent translation of the neutron beam and gamma ray detectors in 15 steps from the bottom to the top of the breast. In addition, at each translation step this setup is rotated at 8 different angles from 0 to $180^{\circ}$. Therefore, a three-dimensional view of the breast volume is obtained. Figure 2 a shows the rotation of the neutron beam around the left breast.

The second geometry has a specific collimator known as a Rotating Modulation Collimator (RMC) (Sharma et al., 2007b). While the RMC rotates, the incident gamma rays are modulated in a manner predicted by its geometry. In this situation, the spatial information of the sample could be obtained without the simultaneous rotation of the neutron beam and the detector.

The neutron source is a conical beam with an energy of 2.5 MeV. In this proposed geometry (p-geometry), the RefMOB and nonRef-MOB phantoms lie in a bed in the prone posture, while the breast is in pendent mode. To eliminate the effects of the bed in neutron attenuation, the bed was filled with air. The best direction for neutron irradiation is parallel with the height of body. This is due to the fact that during the irradiation of one of the breasts, the neutron beam should not pass through the other, and thereby its background remains low. In the upward irradiation (up-irr) from the bottom of the breast, the phantom's brain and eyes are exposed, while in the downward irradiation (down-irr) from the top of the breast, mostly the abdominal organs are exposed. Hence, we chose both irradiation directions (up-irr and down-irr) in order to evaluate the amount of the dose in the two different situations. Figure $2 b$ illustrates the up-irr and down-irr of the neutron beam on the left breast of phantoms in the prone posture.

\subsection{Monte Carlo simulation}

All of the simulations were performed using Monte-Carlo MCNP4C code (Briesmeister, 2000). The dose absorbed by 


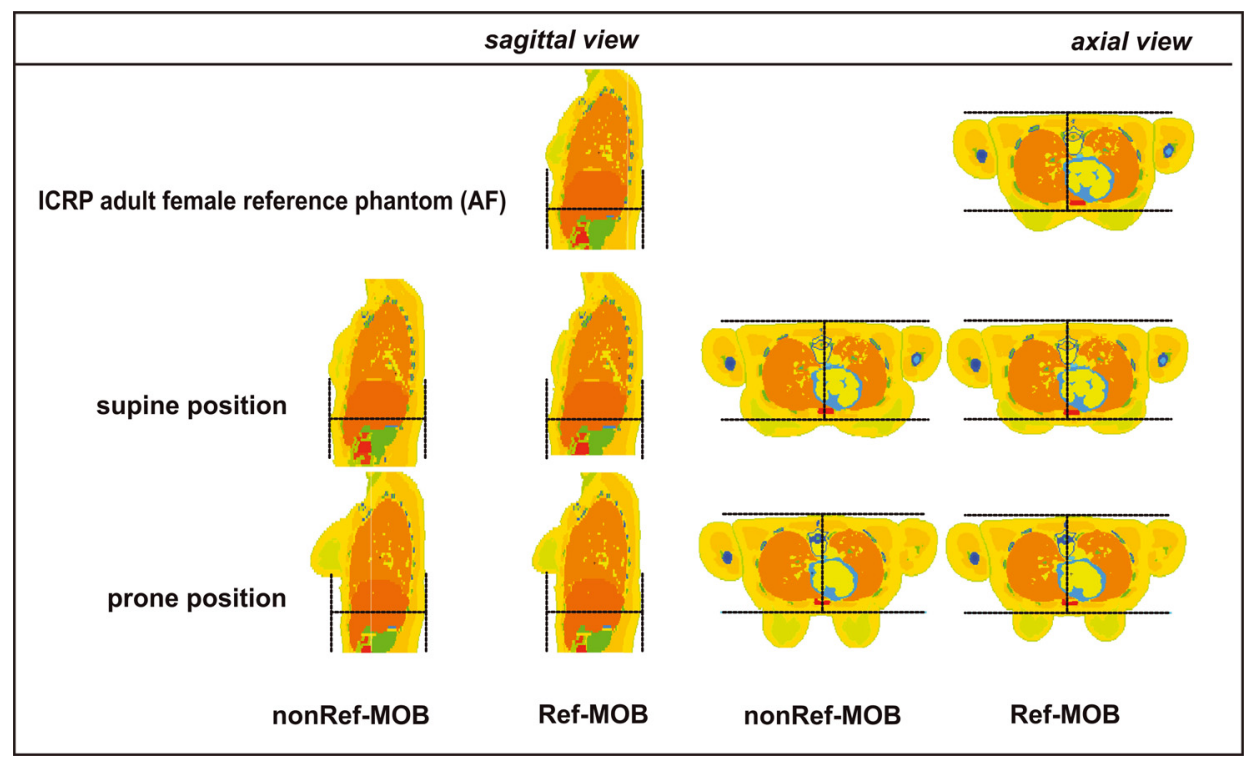

Figure 1. The hybrid voxel model of phantoms including the internal organs in both supine and prone positions. 1) The lines on the right side of the figure (in the axial view of the phantom) show that in the prone posture, the breasts have enough distance from the trunk. It allows illuminating the breasts while the torso is away from the radiation exposure. However, it is not possible in the supine position. The size of the breasts in phantoms with non-reference mass of the breast (nonRef-MOB) can be compared with that in phantoms with a reference mass of the breast (Ref-MOB 2) In the sagittal view of the phantom (left side of the figure), the lines demonstrate the effect of bed compression on abdominal organs in the prone position compared with the supine posture. The abdomen was reformed to get the shape of bed and its internal organs such as the liver were compressed due to this change.

organs due to neutrons and photons was estimated using the heating tally (tally F6). Since the neutron beam has an aperture with a cross-section in the order of $1 \mathrm{~cm}^{2}$ (commonly used in the NSECT method), the source fluence equals the number of particles emitted from the source. Therefore, the amount of the dose was reported in terms of a definite number of neutrons.

The equivalent dose and effective dose were estimated by the weighting factors reported in ICRP Publication 103 (Valentin, 2007). It is worth noting that the definition of the effective dose used in this article was extended and it differs from the sex-averaged recommendation of the ICRP. It is reasonable since the ICRP developed the concept of the effective dose for radiation protection purposes, where the gender is generally unknown, whereas the gender is known in medical radiation applications. It is associated with no limitation, because the AF phantom's organs have the properties that only belong to the reference anatomy of the female gender with no dependency on the male gender. Therefore, the AF specific organ doses can be used with tissue weighting factors separately.

The distribution of the dose in each voxel of the breast was obtained using the mesh tally type 3 . In all simulations, $2 \times$ $10^{9}$ particles were tracked to obtain statistically meaningful results with relative errors less than $5 \%$.

\section{Results}

\subsection{Developing the phantom in the supine and prone postures}

Figure 3 represents the process of developing the phantom in the prone posture. Figure 3 a shows a MRI image series
Table 1. The mass of adipose and glandular tissues (g) of all phantoms in supine and prone postures. Reference and non-reference masses of the breasts are referred to as Ref-MOB and nonRef-MOB, respectively.

\begin{tabular}{lccc}
\hline & AF & nonRef-MOB & Ref-MOB \\
\hline Adipose & 150 & 307 & 148 \\
\hline Glandular & 100 & 111.5 & 93 \\
\hline
\end{tabular}

of the breasts in the supine and prone postures. In Figure 3b, the breasts with initial and reference masses are depicted. The masses of the adipose and glandular tissues before and after modification are tabulated in Table 1. According to the table, the total mass of the volunteer's breast and the mass of her mammary glands is $420 \mathrm{~g}$ and $100 \mathrm{~g}$, respectively. As known, the total mass of the AF's breast is about $250 \mathrm{~g}$, while the mass of its mammary glands is almost $100 \mathrm{~g}$ (Valentin, 2002). Therefore, to adjust the breast mass to that reported in ICRP Publication 89, the volume of adipose tissue was decreased, while the volume of glandular tissue remained constant. In this procedure, the shape of the breast did not change (Fig. 3b). In Figure $3 \mathrm{c}$, the process used to adapt the trunk of the volunteer to that of the AF is indicated.

Figure $3 \mathrm{~d}$ demonstrates the phantom's trunk before and after the modifications. From Figure 3d, the effects of the bed on the interior abdominal organs such as the stomach and liver is observed. Table 2 presents the difference between the organ volumes in the initial and modified modes. The discrepancy in organ volumes was below $18 \%$ for the anterior abdominal tissues, except for the transverse part of the large intestine. 

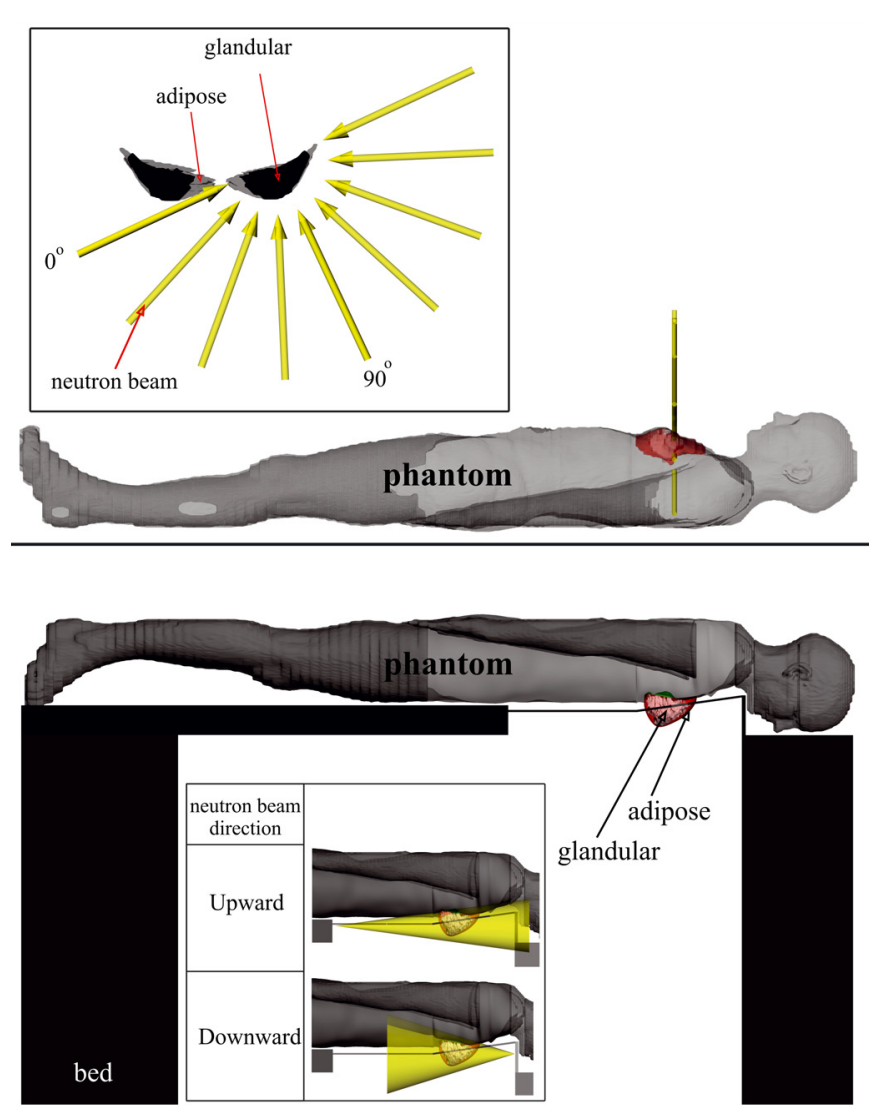

Figure 2. The view of NSECT acquisition geometries: (a) the top of the figure is common geometry (c-geometry). The rotation of the neutron beam in one irradiation step around the left breast was demonstrated at 8 different angles from 0 to $180^{\circ}$. The angular distance between two adjacent rotation angles is $22.5^{\circ}$. (b) The bottom of the figure is a proposed geometry (p-geometry), in which the spatial information of the sample could be obtained without the simultaneous rotation of the neutron beam and the detector due to using a Rotating Modulation Collimator (RMC), and the patient lies on the bed in the prone posture. The direction of the neutron beam is shown. The position of the detectors is not depicted in this figure.

Table 2. Difference (in percent) in the organ volumes before and after modifications.

\begin{tabular}{lc}
\hline Organs & $\begin{array}{c}\text { Difference in organ } \\
\text { volume }(\%)\end{array}$ \\
\hline Transverse colon & 24 \\
Pancreas, colon, gall bladder & $15-18$ \\
Stomach, small intestine, liver, ureters & $10-15$ \\
Other organs & $<10$ \\
\hline
\end{tabular}

\subsection{Dose received by the Ref-MOB phantom}

Table 3 shows the amount of the dose delivered to the RefMOB phantom in two different systems when the left breast is exposed. As expected, the contribution of secondary gamma rays to the dose is about 200 -fold less than neutrons. So, the dose due to neutrons can describe the behavior of the total dose.
It was found from this table that in the c-geometry, the equivalent dose received by adipose tissue is similar to that of mammary glands. In the p-geometry, the equivalent dose of adipose tissue is 1.5-fold higher than glandular tissue in downirr, while this trend is reversed in up-irr. In this measuring system, the equivalent dose of the breast was about 1.44-fold higher than that in the common NSECT configuration.

The distribution of the deposited energy in each voxel of the breast is shown in Figure 4. Figure 4a relates to the cgeometry during the rotation of the beam around the breast. According to the figure, the absorbed dose at the beginning and at the end of the beam trajectory (glandular tissue) is higher than the other parts. The maximum dose received in a voxel of adipose tissue is about 2-fold higher than that in a voxel of mammary glands. The average energy deposition in the breast of the Ref-MOB phantom is $0.5 \mathrm{MeV}$ per emitted neutron. However, the maximum deposited energy is $0.82 \mathrm{MeV}$ per one emitted neutron, which has good agreement with the deposited energy calculated by Bender et al. (2007).

Figure 5 displays the averaged absorbed dose of adipose and glandular tissues in 9 rotation angles at different steps of beam translation. The mammary gland dose is higher than the average dose of total irradiation within -2.7 to $2.7 \mathrm{~cm}$. To assess the dispersion in the absorbed dose data at different positions of the source, the ratio of the standard deviation to the mean value was calculated as the coefficient of variation $(\mathrm{CV})$. The CV of the absorbed dose for glandular and adipose tissues is $17.6 \%$ and $70.82 \%$, respectively.

Figure $4 \mathrm{~b}$ relates to the p-geometry that depicts a sagittal section of the dose distribution. From the figure, energy is not uniformly deposited in the total volume of the breast. The rate of the delivered dose at the beginning of the beam path is high and it decreases gradually as the beam penetrates into the tissue. The CVs of the absorbed dose in down-irr and up-irr are $95.62 \%$ and $63.97 \%$, separately.

In the c-geometry, the maximum delivered dose to adjacent organs is about 3.5-fold less than the value of the breast dose. When the left breast is being bombarded, the heart and lungs receive the maximum amount of the dose, respectively, whereas irradiating the right breast causes a higher amount of the dose to be received by the lungs. In the p-geometry, the highest amount of the dose is received by the heart, which is significantly lower than the breast dose. The organs which are in front of the neutron source, such as the eye lens and brain in up-irr and stomach in down-irr, absorb greater doses than the other organs. In the two systems, increasing the distance from the irradiation area reduces the equivalent dose, so that the absorbed doses of the farther organs of all phantoms are almost the same.

In the c-geometry, the effective dose of the Ref-MOB phantom in a single irradiation is $4.4 \times 10^{-6} \mathrm{mSv}$ per one million incident neutrons. The ratio of the whole-body effective dose to the breast effective dose is lower than 1.5. Since the dominant part of the energy is stored in the breast, the effective doses of the breasts and whole body are similar. The effective dose in the p-geometry is about $12 \%$ more than that in the initial NSECT tomographic system. 


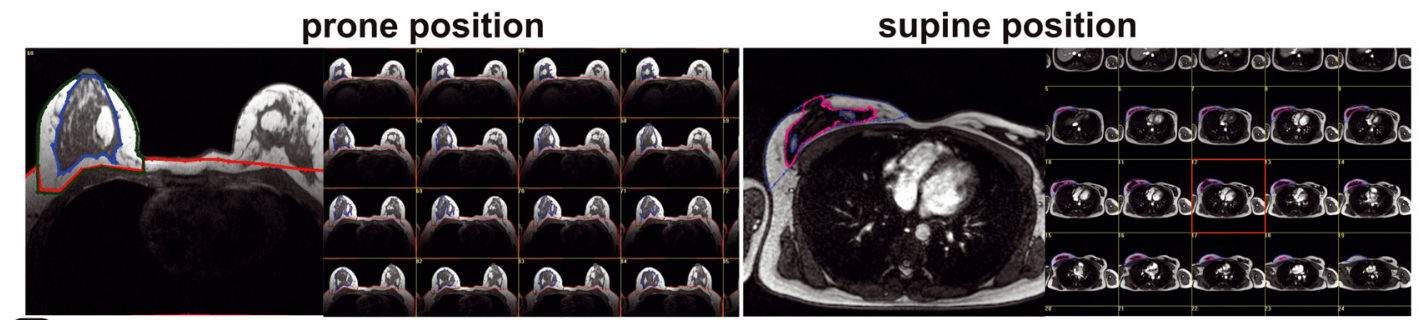

a.
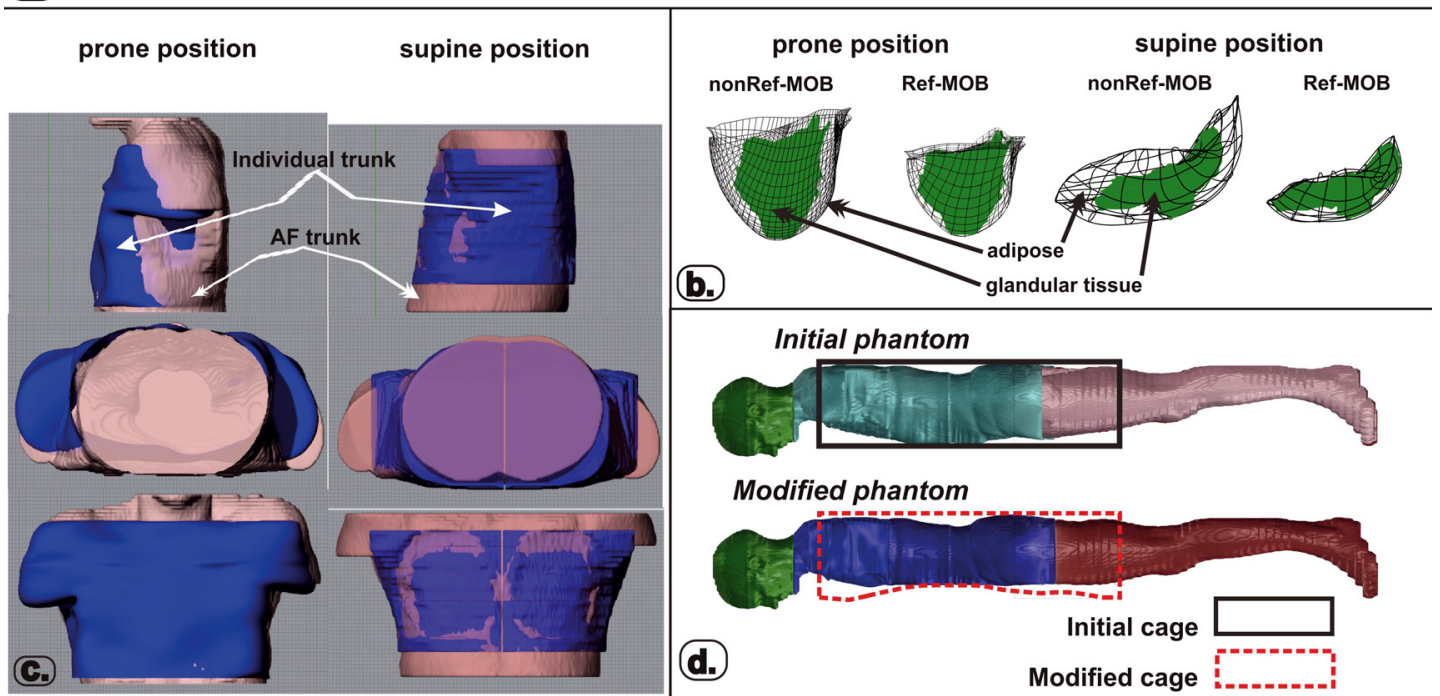

(b.)

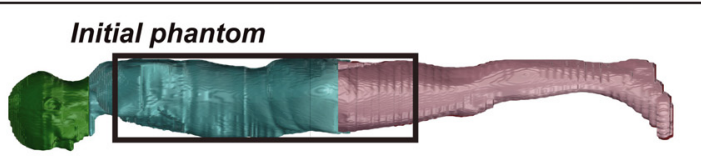

\section{Modified phantom}

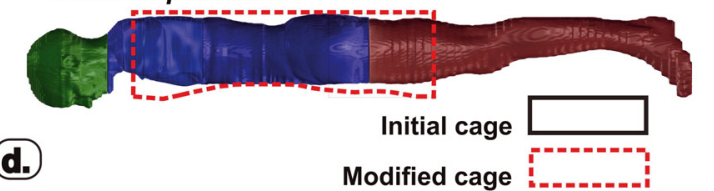

Figure 3. (a) A part of the MR image set from the breasts of a volunteer in the prone and supine positions. In the prone position, a breast matrix coil was used, whereas imaging in the supine posture was done by a body matrix coil; (b) 3D model of the boundary of breast tissues in both positions of the body. The model of non-reference breasts was originally exported from an image set by 3D-DOCTOR's complex surface rendering functions as a polygon mesh model. The polygon mesh of each tissue (glandular and adipose tissues) was then exported to the Rhinoceros software. Finally, the boundary of adipose tissue was scaled by the factor that its volume was matched to the reference value. The glandular tissue remains constant with no scaling due to the similarity of its mass to the AF glandular tissue (c) replacing the subject's trunk with the trunk of the AF. In the prone position, the skin adjacent to the breasts of the phantom was rebuilt as a combination of skin on the back of the AF and the chest of the subject. In the supine position, the skin adjacent to the breasts of the AF was replaced by that of the subject (d) flattening the abdomen of the AF using the "cage" option. First, a box-shaped cage surrounded the torso. Then, the front face of the cage was reformed, and thereby the abdomen was pushed inward.

\subsection{Dose received by the nonRef-MOB phantom}

Table 4 shows the value of the dose for the nonRef-MOB phantom. In the c-geometry, the equivalent doses of adipose and glandular tissues are similar, as observed for the Ref-MOB phantom. The equivalent dose of the breast in the p-geometry is higher than that in the c-geometry by a factor of about 1.77 .

The value of the equivalent dose in the organs located far from the source is similar to that of the Ref-MOB phantom. The organs adjacent to the breast such as the lungs receive the highest amount of the dose. The effective dose of the nonRefMOB phantom is about $3.5 \times 10^{-6} \mathrm{mSv}$ per 1 million incident neutrons in the c-geometry $(31 \%$ lower than that in the p-geometry), while the ratio of the whole-body effective dose to the breast effective dose is lower than 1.5 in the p-geometry, and is 1.1 in the c-geometry.

From Figure 4a, the maximum amount of the dose in a voxel of adipose tissue is about 4-fold higher than that in a voxel of the mammary glands. During the neutron beam displacement, the $\mathrm{CV}$ of the dose for adipose tissue and mammary glands is $21.4 \%$ and $71.34 \%$, respectively, which is similar to those in the Ref-MOB phantom.

\subsection{Comparison between the Ref-MOB and nonRef-MOB phantoms}

Considering Tables 3 and 4, the breast of the nonRef-MOB phantom receives a lower equivalent dose compared with the Ref-MOB phantom. The difference in the dose of the two phantoms is about $25 \%$ in the c-geometry and about $12 \%$ in the p-geometry.

In the c-geometry, the maximum dose delivered to a voxel of adipose tissue is constant in the two phantoms. However, the maximum energy deposited in a voxel of mammary glands in the nonRef-MOB phantom is half that in the Ref-MOB phantom. The average dose of adipose tissue and glandular tissue in the nonRef-MOB phantom is about $36 \%$ and $33 \%$ less than that in the Ref-MOB phantom, respectively.

In the p-geometry, the mammary gland dose of the nonRef breast does not differ from the Ref breast in up-irr; but in 


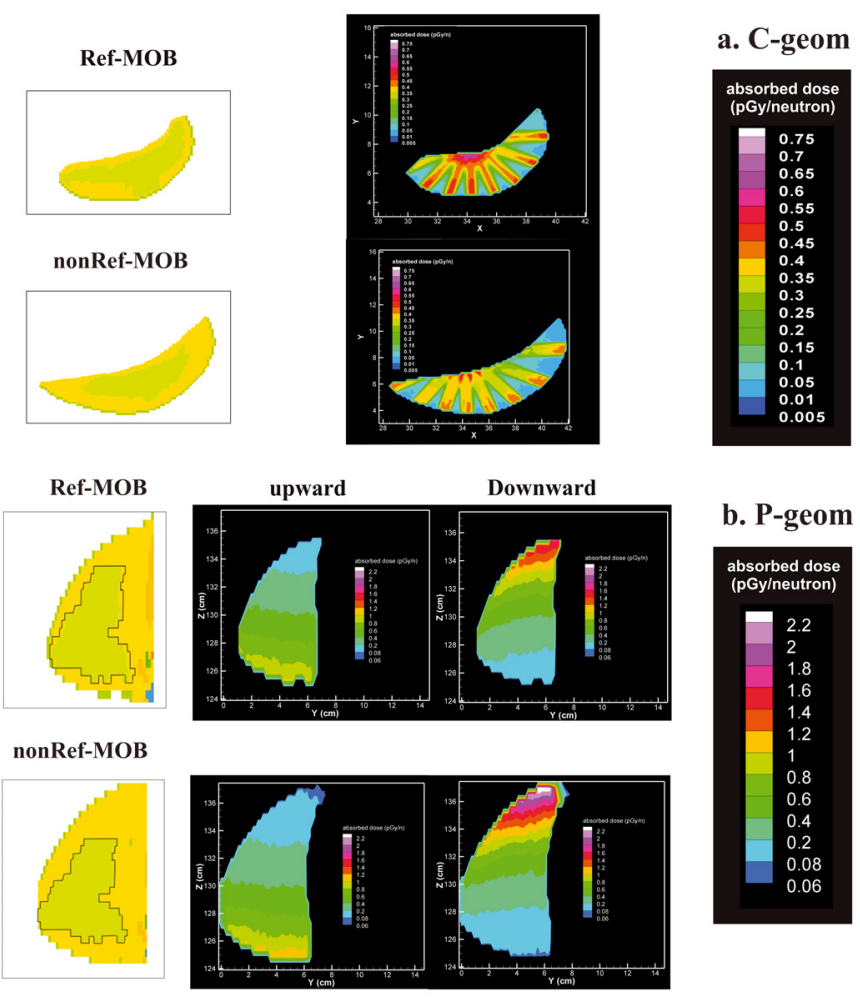

Figure 4. The absorbed dose distribution per mass of a breast voxel: (a) transverse view of phantoms in the supine position, (b) sagittal view of phantoms in the prone posture. Reference and non-reference masses of the breasts are referred to as Ref-MOB and nonRef-MOB, respectively.

Table 3. The amount of the dose delivered to the Ref-MOB phantom in two different geometries when the left breast is exposed $\left(10^{-2} \frac{p S v}{\text { neutron }}\right)$.

\begin{tabular}{lccc}
\hline & \multicolumn{3}{c}{ Tomographic geometry } \\
Classic & $\begin{array}{c}\text { Proposed } \\
\text { down-irr }\end{array}$ \\
Up-irr \\
Organs & \multicolumn{4}{c}{ Equivalent dose } \\
\hline Left breast & 524.34 & 753.23 & 752.57 \\
adipose tissue & 518.8 & 850.19 & 684.15 \\
glandular tissue & 532.54 & 609.32 & 854.13 \\
Right breast & 7.754 & 4.587 & 4.437 \\
Brain & 0.305 & 0.175 & 4.731 \\
Eye lens & 1.228 & 0.602 & 37.478 \\
\hline Lungs & 50.849 & 9.097 & 5.748 \\
Heart & 142.2 & 34.564 & 14.381 \\
\hline Esophagus & 22.792 & 4.21 & 3.551 \\
Thymus & 36.064 & 4.406 & 6.615 \\
\hline Liver & 6.891 & 5.92 & 2.59 \\
Stomach & 11.291 & 13.937 & 4.761 \\
\hline Colon & 0.289 & 0.676 & 0.144 \\
Kidneys & 2.147 & 2.349 & 0.788 \\
\hline Gall bladder & 3.759 & 4.431 & 1.566 \\
Spleen & 10.07 & 4.974 & 2.112 \\
\hline \multicolumn{3}{c}{ Effective dose } \\
\hline The proportion of the breast & 31.9 & 45.39 & 45.35 \\
dose in the effective dose & \multicolumn{3}{c}{} \\
\hline Whole body & 44.39 & 49.81 & 48.62 \\
\hline
\end{tabular}

Table 4. The amount of the dose delivered to the nonRef-MOB phantom in two different geometries when the left breast is exposed $\left(10^{-2} \frac{p S v}{\text { neutron }}\right)$.

\begin{tabular}{|c|c|c|c|}
\hline \multirow[b]{4}{*}{ Organs } & \multicolumn{3}{|c|}{ Geometry } \\
\hline & \multirow[t]{3}{*}{ Classic } & \multicolumn{2}{|c|}{ Proposed } \\
\hline & & down-ir & up-irr \\
\hline & & \multicolumn{2}{|c|}{ Equivalent dose } \\
\hline Left breast & 390.23 & 692.65 & 658.08 \\
\hline adipose tissue & 387.11 & 769.73 & 595.41 \\
\hline glandular tissue & 398.79 & 494.53 & 819.13 \\
\hline Right breast & 6.866 & 6.163 & 6.417 \\
\hline Brain & 0.331 & 0.269 & 2.556 \\
\hline Eye lens & 0.884 & 1.177 & 22.282 \\
\hline Lungs & 45.236 & 9.834 & 6.771 \\
\hline Heart & 119.95 & 31.151 & 17.105 \\
\hline Esophagus & 19.163 & 4.741 & 4.136 \\
\hline Thymus & 27.212 & 6.214 & 7.861 \\
\hline Liver & 6.441 & 5.091 & 3.123 \\
\hline Stomach & 11.115 & 11.275 & 5.529 \\
\hline Colon & 0.308 & 0.546 & 0.212 \\
\hline Kidneys & 2.118 & 2.015 & 0.957 \\
\hline Gall bladder & 3.597 & 3.764 & 1.94 \\
\hline \multirow[t]{2}{*}{ Spleen } & 9.829 & 4.811 & 2.56 \\
\hline & \multicolumn{3}{|c|}{ Effective dose } \\
\hline $\begin{array}{l}\text { The proportion of the breast } \\
\text { dose in the effective dose }\end{array}$ & 23.85 & 41.99 & 39.96 \\
\hline Whole body & 34.96 & 46.17 & 43.11 \\
\hline
\end{tabular}

down-irr, a higher dose (about 23\%) is received by the glandular tissue of the ref-MOB phantom.

\section{Discussion}

\subsection{The initial NSECT geometry}

In the two phantoms, the equivalent dose of the mammary glands is identical to that of adipose tissue. According to Figure 5 , in -2.7 to $2.7 \mathrm{~cm}$, a greater volume of glandular tissue is exposed to fast neutrons, so it absorbs a higher amount of the dose than the average value obtained in total irradiation. Therefore, the energy deposited on mammary glands is higher than on adipose tissue. Outside this range, this trend is reversed. Consequently, these fluctuations lead to the similarity of the dose in the two breast components.

The absorbed doses of the adipose and glandular tissues in the Ref-MOB phantom are higher than in the nonRef-MOB phantom. As high-energy neutrons are irradiated to the breasts, first they are faced with adipose tissue, so they lose energy due to their interactions with adipose elements. Therefore, the maximum energy deposition in both phantoms is similar. On the other hand, more interactions take place between neutrons and some adipose elements such as hydrogen, oxygen and carbon of the nonRef-MOB phantom. This is due to the higher mass of the nonRef-MOB adipose tissue, which is about twofold greater than that of the Ref-MOB phantom. Consequently, as a greater reduction in the energy of neutrons happens, the average energy deposition and average equivalent dose of the nonRef-MOB phantom are less than those of the Ref-MOB. 

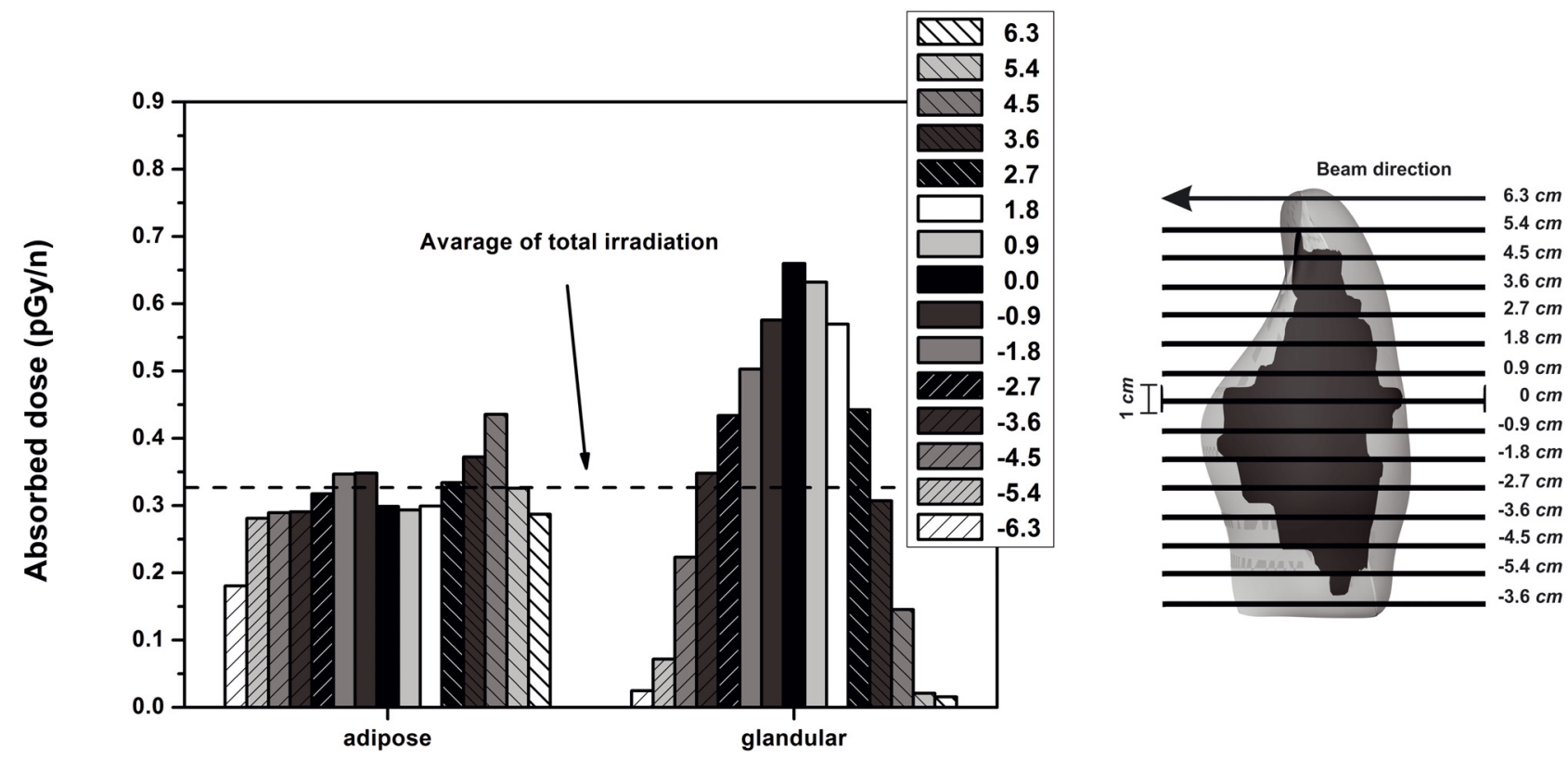

Figure 5. The absorbed dose of adipose tissue and mammary glands of the AF averaged on one step of rotation of the neutron source in different translation steps.

The maximum energy deposited in a voxel of mammary glands is lower than that of adipose tissue. It was indicated that neutron energy decreased due to interaction with the adipose tissue surrounding the glandular tissue. The cross-section of inelastic interaction and neutron capture are high for carbon and hydrogen, respectively. So, adipose tissue can serve as a shield for glandular tissue. Besides, the maximum energy deposition on the mammary glands of the nonRef-MOB phantom is lower than that of the other phantom. The glandular tissue of the nonRef-MOB phantom is encircled by more adipose tissue. Therefore, the dose of the glandular tissue decreases as the adipose tissue volume increases.

To calculate the total amount of the dose in total irradiation during the operation of the NSECT method, the number of emitted neutrons which provides a suitable image resolution for an accurate diagnosis should be determined. It has been established that only $10^{4}$ gamma events are required in the detectors to provide quantitative accuracy of $95 \%$ in tomographic images. According to a study evaluating the efficiency of inelastic scattering, this can be obtained by 10 million incident neutrons (Kapadia et al., 2004). This value is assumed as required incident neutrons in one irradiation state. In the cgeometry, one can provide 15 two-dimensional images from the breast volume. Therefore, considering Tables 3 and 4, the breast equivalent dose per tomogram image is $0.47 \mathrm{mSv}$, which is less than a mammogram image (ICRP, 2001). In total irradiation, the breast equivalent dose is $7.05 \mathrm{mSv}$, which is onethird of the minimum dose delivered to the breast in a typical adult chest CT scan reported in ICRP Publication 87 (Rehani et al., 2000). The total effective dose in the Ref-MOB phantom is $0.6 \mathrm{mSv}$. In the nonRef-MOB Phantom, the breast equivalent dose and effective dose are reduced by factors of 1.34 and 1.26 , respectively. The translation steps cover the breast volume along the body's height, but to provide data from the whole volume of breast, the number of rotation angles should be increased.

\subsection{The proposed NSECT geometry}

Figure $4 \mathrm{~b}$ shows that a greater volume of adipose tissue is located on top of the glandular tissue. Therefore, in up-irr, the shielding effect of the adipose tissue decreases and the mammary glands of both phantoms receive the same dose. Due to the higher mass of the breast in the nonRef-MOB phantom, the shielding effects of adipose tissue on the glandular tissue are enhanced in down-irr. Therefore, it clearly leads to a decrease in the received dose, as was described for phantoms in the previous section.

In Figure 4b, the non-uniformity in the dose distribution is significantly affected by increasing or decreasing the doses of the adipose and glandular tissues. In down-irr, the adipose tissue was exposed to fast neutrons so more energy was deposited in this tissue, while in up-irr, this happened for glandular tissue.

In the p-geometry, the breast absorbs higher amounts of the dose compared with the c-geometry. This is because of the local deposition of energy in the breast in the p-geometry. Since only the breast is directly exposed to the irradiation, doses delivered to the other organs significantly decrease compared with the c-geometry.

To calculate the total value of the dose, it is assumed that in the p-geometry, the exposure time is the same as the cgeometry. In this configuration, despite covering the whole volume of the breast through only one irradiation state, the number of required neutrons providing an appropriate resolution of images is not decreased, due to attenuation of neutrons 
and gamma rays by the patient's bed and RMC collimator, respectively. With this assumption (for $1.35 \times 10^{9}$ incident neutrons), the total equivalent dose of the irradiated breast and total effective dose for the Ref-MOB phantom are evaluated at $10 \mathrm{mSv}$ and $0.68 \mathrm{mSv}$, respectively. The value of the equivalent dose of the breast is less than that in a CT scan of the chest (Rehani et al., 2000). The breast volume also affects the number of required neutrons. For the nonRef-MOB phantom, the total equivalent dose and total effective dose decrease by a factor of 1.087. However, it was expected that more neutrons are required for high-resolution images compared with the breast with lower mass.

It should be noted that, while the high number of incident neutrons causes more resolution in the images of elemental concentration, it leads to a higher received dose. Consequently, the optimum number of neutrons should be selected to have a low delivered dose and high image resolution.

\section{Conclusion}

The NSECT technique, with low-dose screening modalities, can serve for breast cancer diagnosis. Due to the significance of the amount of the dose received by an individual, the dosimetric aspect of this technique was considered in this investigation.

Two models of the human whole body in the supine and prone positions were created to assess the dose in NSECT systems. In the developed phantoms, the volume of some abdominal organs varied by about $20 \%$ compared with the reference volumes of organs reported in ICRP Publication 89. Considering the facts that the organs mentioned were away from the area under exposure and irradiation was local, these variations were negligible. Therefore, their delivered doses were low and did not have a significant effect on the value of the effective dose. However, to design an exact model in the supine and prone positions, developing an Iranian adult female reference phantom in two positions is under investigation.

The results of the current investigation showed that the pgeometry provides a better situation, since breasts are pendent and the absorbed doses of adjacent organs decrease considerably. In two NSECT configurations, the value of the breast equivalent dose per tomogram image was $0.47 \mathrm{mSv}$ neutrons, which was lower than that in mammography. In total irradiation, the breast equivalent dose was less than $10 \mathrm{mSv}$, which is received by the breast in a typical chest CT scan. The results indicate the promising future of the NSECT method as a low-dose tomographic imaging system for breasts. However, the dose delivered to the breast was dependent on the exposure time and the number of incident neutrons. In addition, neutron flux influenced the accuracy of the elemental specification and image resolution. Therefore, to determine the best situation providing the optimum level of dose and image resolution, a simultaneous study of dosimetric and imaging aspects of NSECT is required.

Acknowledgements. The authors thank Mohhamad-Javad Mehrabifar, at Qaem MRI Center, Masshad, for providing MR images required in this study.

\section{References}

Andrási E., Suhajda M., Sáray I., Bezúr L., Ernyei L., Réffy A. (1993) Concentration of elements in human brain: glioblastoma multiforme, Sci. Total Environ. 139, 399-402.

Bender J.E., Kapadia A.J., Sharma A.C., Tourassi G.D., Harrawood B.P., Floyd C.E. (2007) Breast cancer detection using neutron stimulated emission computed tomography: Prominent elements and dose requirements, Med. Phys. 34, 3866-3871.

Bolch W., Lee C., Wayson M., Johnson P. (2010) Hybrid computational phantoms for medical dose reconstruction, Radiat. Environ. Biophys. 49, 155-168.

Briesmeister J. (2000) MCNP 4C General Monte Carlo n-particle transport code, Los Alamos National Laboratory.

Garg A., Singh V., Weginwar R., Sagdeo V. (1994) An elemental correlation study in cancerous and normal breast tissue with successive clinical stages by neutron activation analysis, Biol. Trace Elem. Res. 46, 185-202.

Geraki K., Farquharson M., Bradley D. (2002) Concentrations of Fe, $\mathrm{Cu}$ and $\mathrm{Zn}$ in breast tissue: a synchrotron XRF study, Phys. Med. Biol. 47, 2327-2339.

Grosswendt B. (2012) ICRP Publication 110, Radiat. Prot. Dosim. 150, 124-126.

Hollán S.R. (1997) Transfusion-associated iron overload, Curr. Opin. Hematology 4, 436-441.

Hoseinian E., Rafat-Motavalli L., Miri-Hakimabad H. (2014) Development of 9-month pregnant hybrid phantom and its internal dosimetry for thyroid agents, J. Radiat. Res. 2014, 1-18.

ICRP (2001) Your patient: a guide for medical practitioners. Also includes: Diagnostic reference levels in medical imaging-review and additional advice ICRP Supporting Guidance 2, Ann. ICRP 31.

Kapadia A.J. (2009) Simulations to Evaluate Accuracy and Patient Dose in Neutron-Stimulated, Emission-Computed Tomography (NSECT) for Diagnosis of Breast Cancer: DTIC Document.

Kapadia A.J., Floyd C., Howell C., Harrawood B. (2004) Sampling Requirements for Neutron stimulated emission computed tomography, RSNA, Physics (Digital Imaging, PACS) session.

Kapadia A.J. et al. (2006) Neutron spectroscopy of mouse using neutron stimulated emission computed tomography (NSECT), Nuclear Science Symposium Conference Record, IEEE 6, 3546-3548.

Kapadia A.J. et al. (2008) Neutron stimulated emission computed tomography for diagnosis of breast cancer, IEEE Trans. Nucl. Sci. 55, 501-509.

Mutter J., Naumann J., Schneider R., Walach H. (2007) Mercury and alzheimer's disease, Fortschritte der Neurologie-Psychiatrie, 75, 528-538.

Powell L.W. (2005) Hemochromatosis. In: Harrisons Principles of Internal Medicine, 16th edn. (MacGraw Hill, New York), p. 2298.

Powell L.W., George D.K., McDonnell S.M., Kowdley K.V. (1998) Diagnosis of hemochromatosis, Ann. Intern. Med. 129, 925-931.

Rehani M., Gordon L., Shrimpton P. (2000) ICRP Publication 87: Managing patient dose in computed tomography, Ann. ICRP $\mathbf{3 0}$, $1-45$.

Sharma A.C. et al. (2006) Development of a high-energy gamma camera for use with NSECT imaging of the breast, Nuclear Science Symposium Conference Record, IEEE 6, 3925-3927.

Sharma A.C., Harrawood B., Bender J., Tourassi G., Kapadia A. (2007a) Neutron stimulated emission computed tomography: a 
Monte Carlo simulation approach, Phys. Med. Biol. 52, 6117-6131.

Sharma A.C. et al. (2007b) Design and development of a high-energy gamma camera for use with NSECT imaging: feasibility for breast imaging, IEEE Trans. Nucl. Sci. 54, 1498-1505.

Valentin J. (2002) Basic anatomical and physiological data for use in radiological protection: reference values: ICRP Publication 89, Ann. ICRP 32 (3), 1-277.
Valentin J. (2007) ICRP Publication 103. The 2007 recommendations of the international commission on radiological protection, Ann. ICRP 37.

Xu X.G., Eckerman K.F. (2010) Handbook of anatomical models for radiation dosimetry (Taylor \& Francis, New York).

Yaman M., Atici D., Bakirdere S., Akdeniz I. (2005) Comparison of trace metal concentrations in malign and benign human prostate, J. Med. Chem. 48, 630-634.

Cite this article as: N. Araghian, H. Miri-Hakimabad, L. Rafat-Motavalli. Modeling the adult female phantom in the supine and prone postures and initial dose assessment in breast cancer diagnosis with Neutron Stimulated Emission Computed Tomography. Radioprotection 50(2), 101-109 (2015). 